\title{
Moving Forward Towards a Positive Ethical Healthcare Climate in a Multicultural Context
}

\author{
Adel F Almutairi ${ }^{1,2^{*}}$ \\ ${ }^{1}$ King Abdullah International Medical Research Centre (KAIMRC), Riyadh, KSA \\ ${ }^{2}$ King Saud Bin Abdulaziz University for Health Sciences, Riyadh, KSA \\ *Corresponding author: Adel F Almutairi, King Abdullah International Medical Research Centre (KAIMRC), Riyadh, KSA, Tel: 00966555900336; E-mail: \\ almutairiAd1@ngha.med.sa
}

Rec date: Sep 07, 2015; Acc date: Oct 16, 2015; Pub date: Oct 26, 2015

Copyright: (c) 2015 Almutairi AF. et al. This is an open-access article distributed under the terms of the Creative Commons Attribution License, which permits unrestricted use, distribution, and reproduction in any medium, provided the original author and source are credited.

\begin{abstract}
Many countries around the globe are experiencing a demographic shift in their populations, due to several factors, including immigration, acculturation, mobility of workforces and so forth. These factors have contributed to the creation of multicultural environments characterized by differences in cultures, values, beliefs, ethical and moral perspectives, and languages, as well as historical, political and economic circumstances of their members. In healthcare settings, such diversity and the associated socio-political factors can pose ethical challenges for healthcare providers, and patients and their families alike. Almutairi's model of critical cultural competence (CCC) is a promising approach designed to address the complexities that arise during cross-cultural interactions in healthcare settings. Healthcare settings are imbued with socio-political challenges related to class, racialization, poverty and gender. The ultimate goal of CCC is to provide a positive ethical climate that promotes cultural and physical safety as well as equity in health care environments. CCC approach can influence organizational policy, healthcare professional orientation and continuous professional educational initiatives.
\end{abstract}

Keywords: Cultural safety; Equity; Ethics; Ethical climate

\section{Background}

Many countries around the globe are experiencing a demographic shift in their populations, due to several factors, including colonisation, immigration, acculturation, mobility of workforces and so forth [1]. Although the degree of such demographic changes varies across countries, it has contributed to the creation of multicultural environments characterized by diverse cultures, values, beliefs, ethical and moral perspectives, and languages, as well as historical, political and economic circumstances of their members.

In healthcare settings, many studies in the literature-conducted in different countries and settings, including developed and developing countries-have demonstrated the difficulties that healthcare providers, patients and their families experience during cross-cultural interactions [2-9]. Culture in itself is not a problem; it represents valuable heritages that enrich any society and provide lenses for viewing situations from alternative perspectives. However, many of the factors associated with cultural and linguistic differences are reported to be responsible for forming and shaping such a profound problem, including limited awareness of the meaning of cultural differences and the conceptualization of culture; ethnocentric viewpoints; and inadequate educational preparation [2]. Healthcare settings are also imbued with socio-political challenges related to class, racialization, poverty and gender. These factors can sometimes lead to cultural clashes, misunderstandings in communication and behaviour, discrimination and individual power differences, which in turn could lead to healthcare disparities and inequities [10]. The impact could also go beyond that to avoidance of seeking healthcare services, $[11,12]$ and serious consequences could result.
The question addressed in this paper relates to how we manage these difficulties and challenges whilst providing an ethical climate that is attentive to different perspectives. This paper therefore outlines a conceptual link between cultural diversity and ethical perspectives in healthcare settings. This paper proposes Almutairi's model of critical cultural competence (CCC) as a promising approach that can address the many complexities that arise during cross-cultural interactions [13]. CCC approach can possibly influence organizational policy, healthcare professional orientation, and continuous professional educational initiatives. Figure 1 below provides an overview of the components that constitute this model.

\section{Ethical Challenges in a Multicultural Context}

Establishing the conceptual understanding between socio-cultural factors as a result of diversity among nations, and ethical perspectives is crucial to fully understanding the magnitude of the associated ethical complexity and resultant consequences. As discussed previously, socio-cultural and linguistic differences associated with power determinants complicate cross-cultural interactions in healthcare settings $[2,4,5]$. They often compromise the ethical principle of patients' societal rights in all aspects, including the right to healthcare resources, the right to healthcare knowledge, the right to protection from harm, the right to autonomy, and ultimately the right to equitable healthcare and outcome $[14,15]$. These general but essential aspects of patients' rights that were developed in the 1960s based on recognition of the inherent dignity of human beings 15 are similar in all countries in terms of their purpose and outcome, but often vary in terms of their approaches depending upon the prevailing socio-cultural norms. 
Page 2 of 5

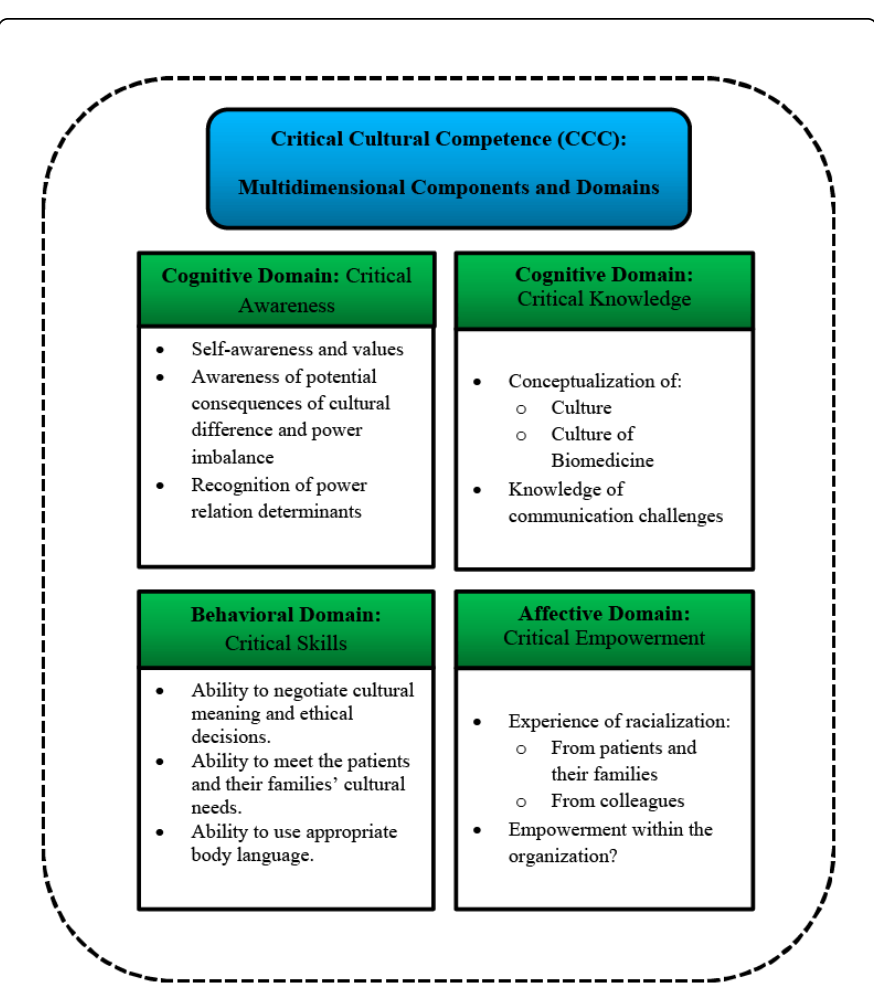

Figure 1: Illustration of multidimensional components of CCC (reproduced with permission from the author) [13].

One way of fulfilling patient rights in a multicultural context is through effective communication, which as a principle ensures a process of exchanging messages accurately and understanding one another's perspectives when executed properly. 10 Without effective communication, nurses and other healthcare providers are unlikely to understand and meet patients' needs in terms of knowledge or resources related to cultural, social, or religious factors. 2 Moreover, healthcare providers will most unlikely be unable to provide the necessary health education, information on disease complexity and treatment plans, and psychological support requirements of their multicultural patients' perspectives [16,17]. Communication challenges have also been shown to compromise patients and families' right to safe healthcare. Many studies in the literature illustrate the risks of ineffective communication during the clinical encounter and how it is associated with healthcare inequities and patient safety $[10,17,18]$. Almutairi also highlighted similar risks in a recent qualitative study, 19 which investigated the cultural competence of a multicultural nursing workforce working in a Saudi hospital in Riyadh city. In this study, twenty-four non-Saudi nurses, coming from more than ten different countries, were individually interviewed. The findings indicated that socio-cultural and linguistic challenges have an impact on patients' cultural, physical, mental, spiritual, and social safety and well-being.

Another complex issue which presents in a multicultural environment and often challenges healthcare providers is the use of non-verbal communication (like inadvertent sign language) which can complicate interaction, as many signs and gestures are not culturally common, shared or acceptable and in some circumstances can be interpreted as offensive [19]. For example, the following interview segment which was drawn from Almutairi's study [19]. With a multicultural nursing workforce in Saudi Arabia and explains how the nurses in this study committed culturally unacceptable actions and running the risk of giving offense:

\section{Research participant}

One time when I was feeding [a] patient ... I was feeding him with my left hand, he got angry with me ... he said la la la' [no, no] I said why? [he said ] It should not be the left hand, it should be the right hand, so from there I've learned a lesson.

Another nurse participant in Almutairi's study19 shared her sentiments about her strong and deep belief in the importance of providing caring touches to her Saudi patients regardless of the patients' cultural perspectives:

\section{Research participant}

Ok for us, if there is somebody you care for, you say 'how are you?' you touch the hand, 'how are you mama or baba? Are you ok today?' Then they will respond, 'I'm fine.' But if you tell somebody that if I'm talking to baba I must touch him, it's not allowed, like I cannot shake your hand [male person] ... I usually talk to my patients and say 'how are you?' And touching them, showing that you are caring for them, you are a lovely person ...

This example of a nurse's belief about expressing care through touch is another example where it might be perfectly appropriate from cultural perspective, but in another context it commits an unconscious cultural transgression. In Western nursing practice this behaviour is used as an acceptable gesture of providing comfort, especially at times of patient and family distress, and of enhancing the nurse-patient relationship; [20] however, this is not necessarily the case in other healthcare contexts or cultures. Non-verbal communication in healthcare settings requires some cultural knowledge obtained through the process of ascertaining and negotiating different cultural meanings. Body language and gestures are culturally specific and their meanings can differ from one culture to another [21-24].

One of fundamental patient right principle is the right to privacy whilst in the hospital, in regards to both their medical information/ records and their physical space. This principle can be challenging in a multicultural environment, particularly when it comes to patients' private physical space and can be easily breached due to different understandings of the physical privacy boundaries amongst different cultures. For example, a number of studies in the literature illustrate the broad definition of privacy in many Muslim communities and how many male and female Muslims value modesty in terms of dress and do not undress in front of others, as they would become extremely embarrassed if asked to [25-27]. These studies also emphasized how some Muslim women avoid unnecessary uncovering even in a hospital environment. Gender-specific care also poses many challenges when it is not properly addressed and negotiated with patients, partly because it can risk the clinical safety of patients. For example, a Western nurse participating in Almutairi's study [19] stated:

\section{Research participant}

There's quite few Saudi, well I'm working on a male ward, so male Saudis who don't want a female nurse to be anywhere near their private parts ... and sometimes we need to be near the private parts so, sometimes we just have to improvise so if you need to take the tube out 
of that area you just have to sort of go in blind and sort of hold the sheet over it so you can't see anything...

One legal concept which is strongly linked to patient rights in healthcare is patient advocacy [15]. This concept, as an inherent and natural part of all healthcare professionals practice but especially nursing, is related to an ethical obligation to advocate for patients' rights and protect them from incompetence or unethical practice [15]. There are many factors that can negatively influence healthcare providers' efforts to fulfil their advocacy role in a multicultural environment. These factors fall beyond the individual agency of the providers and are strongly related to their mediating contexts [10]. They are mainly related to the racialization and disempowerment of healthcare providers caused by economic circumstances, gender, culture, language barriers, geographic location, or institutional or individual racism. In Almutairi's study, [19] lack of confidence and an inability to advocate for patients or challenge safety concerns and confront discrimination were amongst indicators of multicultural nurses' disempowerment.

The concept of disempowerment can led to another pressing ethical issue in a multicultural healthcare context which can have a profound impact on a provider's performance, and ultimately on healthcare outcomes. The issue arises when the healthcare providers are unable to follow certain moral beliefs in given situations and are consequently unable to take the correct action due to political or social constraints. As a result, the healthcare provider may experience a psychological disturbance called moral distress [28-31]. According to the American philosopher Jameton, [32] who coined this term, moral distress occurs "when one knows the right thing to do, but institutional constraints make it nearly impossible to pursue the right course of action" (p. 6). Although the literature identifies many of these contextual issues as political, practical, or ethical, there has been little attention devoted to the link between cultural conflicts and moral distress.

This link however was intensely observed in many multicultural nurses' experiences in Almutairi's Saudi Arabian study [19]. The qualitative component of his study illuminated the moral distress that the nurses suffered as a result of racialization and disempowerment or socio-cultural and linguistic conflicts either among themselves or with the population that they came to serve. The following interview excerpt serves as one specific example of the ethical issues frequently operative in a multicultural context:

\section{Research participant}

Work ethic is a very different thing [here]. Like our unit assistants never show up to work on time and things like that ... I mean at home you just get fired for that, you do it 2 or 3 times and you get fired, but here they do it all the time and nobody seems to care, and they wander off and go for a cigarette ... and go see their friend in another ward and then they are never here, and nobody seems to mind.

This nurse participant experienced deep offense at the frequent absence of the interpreter, who faced no repercussions from her line manager or employer and left her feeling unsupported and disabled from challenging such behaviour. Realization of the essential role that the interpreter plays in maintaining and promoting the quality of healthcare, as well as protecting patient and nurse safety contributed to increasing her feelings of distress and frustration.

In addition to the above ethical issues that operate in multicultural healthcare contexts, socio-cultural differences often contribute to another complex issue related to culturally-safe ethical decision- making. In many clinical situations, making an ethical decision for a patient can be challenging and difficult demanding much consideration. This becomes even more complex when language barriers and cultural conflicts exist, especially those associated with ethnocentrism, negative attitudes, essentialisation, inaccurate interpretation of individual differences, and inadequate understanding of the deep cultural meaning around the decision [10]. Dealing with these factors could push healthcare providers to impose their own cultural perspectives and beliefs, whether consciously or unconsciously, on their patients during the ethical decision-making process [19]. For example, one aspect of the patient bill of rights refers to understand the complexity of the disease and treatment plan; however this right can be challenging to exercise or fulfil in many complex multicultural situations. In some cultures, family members often conceal the state of the disease from their loved ones to spare them pain, or they want to deliver this kind of information in a cultural or religious template that helps to reduce the intensity of any shocking news.

\section{The Critical Cultural Competence (CCC) Approach}

CCC is a promising approach that can contribute positively to managing the difficulties that healthcare providers experience in a multicultural context. The ultimate goal of CCC is to provide a positive ethical climate that promotes cultural and physical safety as well as equity in healthcare environments, and eventually improving healthcare outcomes for patients, families, and the community. Significantly, the CCC approach has been shown to overcome the limitations of other cultural competence frameworks proposed in the literature, some of which ran the risk of essentialising people and treating cultures as constant facts [10]. In addition, many of the existing frameworks focused on the individual agency to achieve a given competence level. Almutairi and Rodney10 claim that, although the individual agency is a crucial component toward equitable, ethical, and safe healthcare, it represents only one facet of a complex process. It is often difficult to isolate individual agency from its mediating context, as the two are constantly intersecting during interaction process, and the socio-cultural context itself can be viewed as either enabling or constraining. The CCC approach thus takes into account both the individual agency of healthcare provider and contextual factors that could lead to providers' disempowerment and thus posing ethical challenges that could possibly threaten the well-being of both patient and provider alike.

From my theoretical perspective, I conceptualise CCC as a continuous process and infinite learning endeavour that requires the healthcare provider to strive constantly toward providing culturally and ethically safe care and interaction, coupled with policy guidance that addresses the socio-cultural determinants of health and support at the organisational level. According to Almutairi and Rodney, [10] CCC is composed mainly of four multifaceted components, namely, critical awareness, critical knowledge, critical skills, and critical empowerment. These components fall into different conceptual domains; the first two represent the cognitive domain, while the third and fourth represent the behavioural and affective domains, respectively. Significantly, the development process of this approach and the conceptualisation of each of these components were all based on empirical research, which were then theoretically refined through the lens of other influential theories such as cultural safety and postcolonial perspectives $[10,13]$. 
Crucially, the various components of CCC are interrelated and interactive, and one can never operate in isolation from the others. Critical awareness makes up the first essential component in this process, as the view is that its absence would complicate the cultural interaction and negatively affect the peace, equity, and well-being of all people involved. It refers specifically to the recognition of sociocultural differences in a multicultural environment, one's own attitudes and assumptions, and the associated potential complications during cross-cultural interactions. It also includes the recognition of powerrelation determinants such as class, racialization, gender, culture, geographic location, and poverty that often operate in a multicultural context and influence relationships and interactions [13]. The conceptualization of this term has multiple facets because the cultural heritages of people are constructed within their unique socio-political, historical, and the economical contexts that influence the way they interpret the world around them and the way they relate to others. Those contexts also shape people's own ethical views/frameworks and how they conceptualise health and illness. The different cultural lenses, (shaped by their norms, values, practices, behaviours, religion, and language), that people bring into clinical settings could possibly lead to tensions, conflicts, and discrimination. Thus, critical awareness can help healthcare providers to avoid ethnocentric views and cultural impositions which may negatively impinge on the cultural, physical, mental, spiritual and social safety, and well-being [10,33,34].

Critical knowledge comprises the second essential component of the CCC approach. It mainly involves obtaining critical knowledge about the way I conceptualise culture, the risk of relying on a static view of culture, and the dominant cultural model of Western biomedicine, as well as the potential communication challenges. From my perspective, I conceptualise culture as "a fluid and dynamic process that is changing constantly over time based on human interactions. Culture also includes individualized as well as common values, beliefs, and meaning systems that are fundamental to individuals' understandings of their selfhood as a result of particular social, historical and political circumstances" [13]. Following the development of critical awareness and knowledge, critical skills are required. This component involves "an individual's agency to enact the aspects of critical awareness and knowledge when interacting with people from other cultures in the context as a whole" [13]. It also comprises the capacity of the healthcare provider to use intercultural communication skills and appropriate body language and the creation of a space to negotiate different cultural perspectives, and ethical issues and decisions related to care pathways. The final component is critical empowerment, which "encompasses the perception of healthcare providers about their own empowerment within their context, particularly in terms of whether they experienced racialization, or being disempowered due to their gender, economic situation, culture, geographic location or institutional or individual racism" [13].

The development of CCC components at both individual and organisational levels can contribute significantly to the creation of an ethically and culturally safe environment. Patients' rights and cultural, ethical, and social needs as well as healthcare providers' ethical, moral, and cultural safety can be maintained and protected.

\section{Implications and Conclusion}

The difficulties that patients, families and providers encounter due to socio-cultural and linguistic differences in contemporary healthcare settings require specific attention and management. The CCC approach was developed to help manage these difficulties and assist in provision of an ethical, moral and safe climate that attends to patients' and families' rights as well as protects healthcare providers' ethical and cultural safety. CCC is a process and framework which allows healthcare providers to recognise the barriers to quality healthcare, such as ethnocentric viewpoints, prejudices, biases, limited awareness of cultural differences, power relation determinants, essentialism, inadequate knowledge of the conceptualization of culture, and ineffective communication. Providers also need to aware that culture can also be created during a clinical encounter with a patient through the process of negotiating differing deep cultural meanings related to health, illness and ethically sensitive issues. CCC also requires critical skills to enact understandings of critical awareness and knowledge, improving effective communication.

Managers and leaders at the organisational level play a role in this process as they are required to provide continuous support that addresses the factors that inadvertently lead to the disempowerment of healthcare providers in a multicultural environment, such as racialization, racism and discrimination. Specifically, organisations that have a multicultural workforce would benefit from using the CCC framework and approach to guide their policy initiatives, induction and orientation programs and continuous professional development. In addition, organisations that hire foreign educated providers should provide a pre-departure orientation guided by features of the CCC framework and approach to psychologically and intellectually prepare these providers before exposure to the new culture and healthcare setting. Almutairi19 claims that the naïve immersion of providers into new socio-cultural contexts can sometimes cause psychological and emotional disturbances, (such as anxiety, insecurity and depression), which consequently influence their performance in providing ethical and safe healthcare.

\section{Acknowledgement}

I would like to the thank King Abdullah International Medical Research Centre for providing unlimited support during my research project.

\section{References}

1. World Health Organization. Nursing and midwifery services: Strategic directions 2011-2015. Geneva, Switzerland: Authors; 2010.

2. Almutairi AF, McCarthy A, Gardner G (2015) Understanding cultural competence in a multicultural nursing workforce: Registered nurses' experience in Saudi Arabia. Journal of Transcultural Nursing 26:16-23.

3. Almutairi AF, Gardner G, McCarthy A (2013) Perceptions of clinical safety climate of the multicultural nursing workforce in Saudi Arabia: A cross- sectional survey. Collegian journal: The Australian Journal of Nursing Practice Scholarship and Research 20:187-194.

4. Nielsen B, Birkelund R (2009) Minority ethnic patients in the Danish healthcare system - a qualitative study of nurses' experiences when meeting minority ethnic patients. Scandinavian Journal of Caring Sciences 10:1-7.

5. Høye S, Severinsson E (2008) Intensive care nurses' encounters with multicultural families in Norway: an exploratory study. Intensive Crit Care Nurs 24: 338-348.

6. Xu Y (2007) Strangers in strange lands: a metasynthesis of lived experiences of immigrant asian nurses working in Western countries. ANS Adv Nurs Sci 30: 246-265.

7. Lovering S (2006) Cultural attitudes and beliefs about pain. J Transcult Nurs 17: 389-395. 
8. Vydelingum V (2006) Nurses' experiences of caring for South Asian minority ethnic patients in a general hospital in England. Nurs Inq 13: 23-32.

9. Cioffi J (2005) Nurses' experiences of caring for culturally diverse patients in an acute care setting. Contemp Nurse 20: 78-86.

10. Almutairi AF, Rondney P (2013) Critical cultural competence for culturally diverse workforces: toward equitable and peaceful health care. ANS Adv Nurs Sci 36: 200-212.

11. Bernstein J, Bernstein E, Dave A, Hardt E, James T, et al. (2002) Trained medical interpreters in the emergency department: effects on services, subsequent charges, and follow-up. J Immigr Health 4: 171-176.

12. Papps E, Ramsden I (1996) Cultural safety in nursing: the New Zealand experience. Int J Qual Health Care 8: 491-497.

13. Almutairi AF, Dahinten VS, Rodney P (2015) Almutairi's Critical Cultural Competence model for a multicultural healthcare environment. Nurs Inq.

14. Silva MC, Ludwick R (1999) Interstate Nursing Practice and Regulation: Ethical Issues for the 21st Century. Online Journal of Issues in Nursing 4.

15. Lamb M, Storch JL (2013) A hitorical perpective on nursing and nursing ethics. In: Storch JL, Rodney P, Starzomski R, eds. Toward a moral horizon: Nursing ethics for leadership and practice. Don Mills, Ontario: Pearson Canada Inc.

16. Murphy K, Clark JM (1993) Nurses' experiences of caring for ethnicminority clients. J Adv Nurs 18: 442-450.

17. Divi C, Koss RG, Schmaltz SP, Loeb JM (2007) Language proficiency and adverse events in US hospitals: a pilot study. Int J Qual Health Care 19: 60-67.

18. Tuohy D, McCarthy J, Cassidy I, Graham MM (2008) Educational needs of nurses when nursing people of a different culture in Ireland. Int Nurs Rev 55: 164-170.

19. Almutairi AF (2012) A case study examination of the influence of cultural diversity in the multicultural nursing workforce on the quality of care and patient safety in Saudi Arabian hospital [Doctoral dissertation]. Brisbane, Queensland, Queensland University of Technology.

20. Lampley TM, Little KE, Beck-Little R, Xu Y (2008) Cultural competence of north Carolina nurses: A journey from novice to expert. Home Health Care Management Practice 20: 454-461.

21. Fatahi N, Mattsson B, Lundgren SM, Hellström M (2010) Nurse radiographers' experiences of communication with patients who do not speak the native language. J Adv Nurs 66: 774-783.
22. Huang YL, Yates P, Prior D (2009) Accommodating the diverse cultural needs of cancer patients and their families in palliative care. Cancer Nurs 32: E12-21.

23. Hultsjö S, Hjelm K (2005) Immigrants in emergency care: Swedish health care staff's experiences. Int Nurs Rev 52: 276-285.

24. Jirwe M, Gerrish K, Keeney S, Emami A (2009) Identifying the core components of cultural competence: findings from a Delphi study. J Clin Nurs 18: 2622-2634.

25. Halligan P (2006) Caring for patients of Islamic denomination: Critical care nurses' experiences in Saudi Arabia. J Clin Nurs 15: 1565-1573.

26. Cortis JD (2004) Meeting the needs of minority ethnic patients. J Adv Nurs 48: 51-58.

27. Kulwicki AD, Miller J, Schim SM (2000) Collaborative partnership for culture care: enhancing health services for the Arab community. J Transcult Nurs 11: 31-39.

28. Rodney P, d'Agincourt-Canning L, McPherson G, et al (2013) Working within the landscape: Ethics in Practice. In: J. L. Storch, Rodney P, Starzomski R, eds. Toward a moral horizon: Nursing ethics for leadership and practice. Don Mills, Ontario: Pearson Canada Inc.

29. Allen R, Judkins-Cohn T, deVelasco R, Forges E, Lee R, et al. (2013) Moral distress among healthcare professionals at a health system. JONAS Healthc Law Ethics Regul 15: 111-118.

30. 30. Hamric AB, Borchers CT, Epstein EG (2012)Development and testing of an instrument to measure moral distress in ealthcare professionals. AJOB Primary Research 3: 1-9.

31. Lu“tze'n K, Blom T, Ewalds-Kvist Ba, Winch S (2010) Moral stress, moral climate and moral sensitivity among psychiatric professionals. Nursing Ethics. 17: 213-224.

32. Jameton A (1984) Nursing practice: The ethical issues. Englewood Cliffs, NJ: Prentice-Hall.

33. Taylor R (2005) Addressing barriers to cultural competence. J Nurses Staff Dev 21: 135-142.

34. Campinha-Bacote J (2002) The Process of Cultural Competence in the Delivery of Healthcare Services: a model of care. J Transcult Nurs 13: 181-184. 